\title{
Current and Emerging Biologic Therapies for Asthma and COPD
}

\author{
Michael E Wechsler MD MMSc
}

\author{
Introduction \\ Current Approaches to Asthma and COPD Management \\ Approaching Severe Asthma and COPD \\ Personalizing Asthma and COPD Management With Novel Biologic Therapies \\ Current Targets \\ IgE \\ IL-5 \\ Anti-IL-5 and COPD \\ Future Targets \\ IL-4/IL-13 \\ Thymic Stromal Lymphopoietin and IL-33 \\ Non-Th2 Inflammation Targets \\ IL-17 \\ CXCR2 and CCR3 \\ Tyrosine Kinases \\ Summary and Future Directions
}

\begin{abstract}
Historical treatments for asthma and COPD have primarily focused on addressing the underlying inflammation and bronchoconstriction that result in air flow obstruction symptoms, including shortness of breath, cough, chest tightness, and mucus production. However, in the past several years, new research into the underlying pathophysiology of asthma and COPD has led to novel targeted therapies that address the underlying pathways that cause these obstructive disorders. As we have gained a better understanding of underlying disease mechanisms, we have begun to use biomarkers and endotypes to personalize our approach to therapy. Targets for asthma and COPD include immunoglobulin E, interleukin 5, interleukin 4/interleukin 13, thymic stromal lymphopoietin, interleukin 17, tyrosine kinases, and others. The new biologics are generally safe and well tolerated, and are bringing promise and hope of personalized therapy to patients with severe asthma. Key words: asthma; COPD; biologics; IL-5; IL-4/IL-13; TSLP; nonTh2; imatinib. [Respir Care 2018;63(6):699-707. (C) 2018 Daedalus Enterprises]
\end{abstract}

\section{Introduction}

Asthma and COPD continue to affect millions of individuals annually, with millions of emergency department

Dr Wechsler is affiliated with the Cohen Family Asthma Institute, National Jewish Health, Denver, Colorado.

Dr Wechsler discloses relationships with AstraZeneca, Boehringer Ingelheim, Genentech, GlaxoSmithKline, Novartis, Regeneron, Sanofi, and Teva. visits, hundreds of thousands of hospitalizations, and thousands of deaths annually. ${ }^{1}$ Historical treatments primarily focused on addressing the underlying inflammation and bronchoconstriction that result in the air flow obstruction symptoms, including shortness of breath, cough, chest tight-

Dr Wechsler presented a version of this paper at the 56th RESPIRATORY CARE Journal Conference, Respiratory Medications for COPD and Adult Asthma: Pharmacologic Actions to Clinical Applications, held June 2223, 2017 in St Petersburg, Florida. 
ness, and mucus production. However, in the past several years, new research into the underlying pathophysiology of asthma and COPD has led to novel targeted therapies that address the underlying pathways that cause these obstructive disorders (Table 1).

\section{Current Approaches to Asthma and COPD Management}

Current asthma guidelines, including those developed by the National Asthma Education Prevention Program ${ }^{2}$ and the Global Initiative for Asthma, ${ }^{3}$ recommend a stepwise approach to management, with initiation of inhaled corticosteroids in patients with mild persistent asthma, with increased inhaled corticosteroids dosing; the addition of long-acting $\beta$ agonists; leukotriene modifiers, including montelukast or zileuton; and long-acting anticholinergics, for example, tiotropium, to those who remain poorly controlled. For COPD, guidelines recommend initiation of long-acting $\beta$ agonists with or without inhaled corticosteroids, or long-acting anticholinergics, and, in severe cases, the addition of phosphodiesterase inhibitors, including roflumilast. ${ }^{4}$ For some patients with asthma or COPD that fail to respond to these therapies, likely due to poor adherence or underlying disease heterogeneity, oral corticosteroids are often used. However, these are associated with significant systemic toxicity, including adrenal insufficiency, weight gain, hypertension, cataracts, glaucoma, and osteoporosis. Thus, a major question is what to do for those patients who continue to have symptoms or exacerbations despite triple therapy with inhaled corticosteroids, long-acting $\beta$ agonists, and long-acting antimuscarinics or other controllers.

\section{Approaching Severe Asthma and COPD}

The first step in managing patients with severe asthma and COPD involves confirming the diagnosis, assessing adherence, evaluating environmental factors that could be contributing to disease manifestations (eg, environmental allergens or smoke exposure), and investigating comorbid disease that could be contributing to symptoms (eg, gastroesophageal reflux disease, sinusitis, chronic respiratory tract infections, obstructive sleep apnea, or vocal cord dysfunction). ${ }^{5}$ Next, it is important to characterize the type of disease by phenotyping and endotyping the patient. 6 Phenotype refers to the observable characteristics of a specific

Correspondence: Michael E Wechsler MD MMSc, the Cohen Family Asthma Institute, National Jewish Health, Denver, CO 80206. E-mail: WechslerM@NJHealth.org.

DOI: $10.4187 /$ respcare.06322

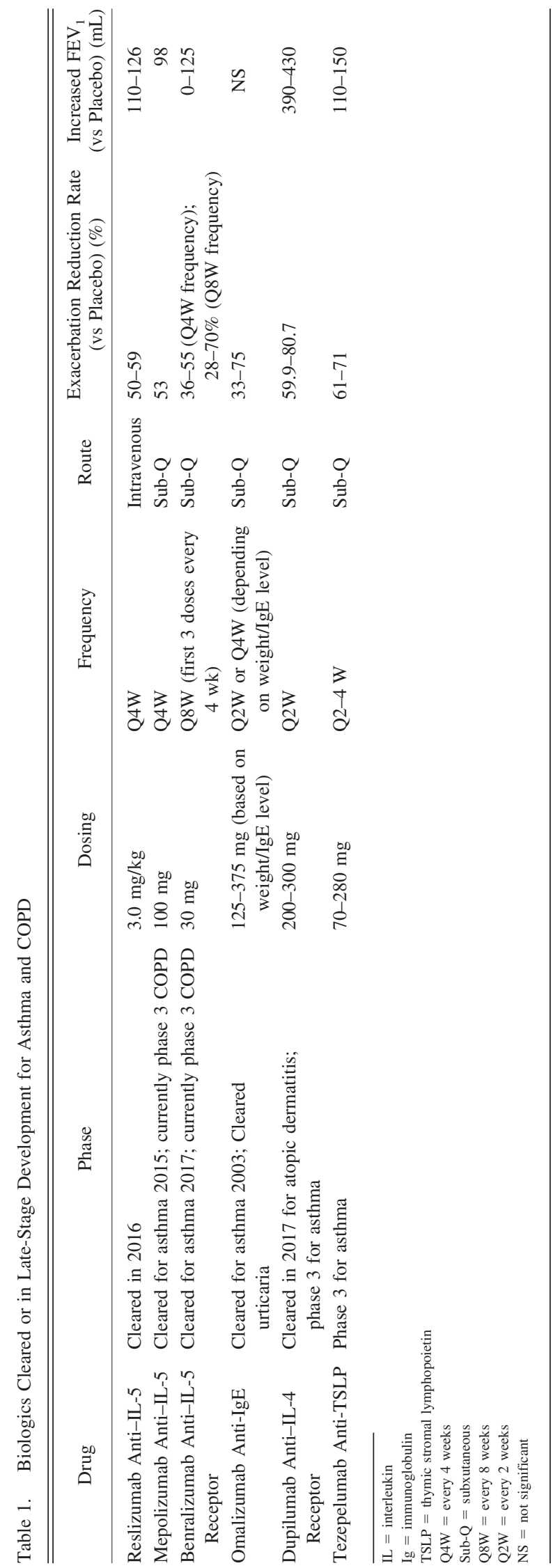




\section{Biologic Therapies for Asthma AND COPD}

disease entity, such as age, sex, race, environmental or allergic exposures, and time of disease onset. Endotype refers to the mechanism of the underlying disease as it relates to cellular, cytokine, or mediator involvement (eg, eosinophilic COPD, immunoglobulin [Ig] E-mediated asthma, interleukin [IL] 5-mediated asthma). Endotyping depends on the use of biomarkers that reflect an underlying disease mechanism. Currently used biomarkers include eosinophils in the blood or sputum, exhaled nitric oxide, IgE levels, and specific cytokines in the blood or sputum. By phenotyping and endotyping patients with asthma and COPD, one can personalize approaches to treatment for those patients who need add-on therapies.

\section{Personalizing Asthma and COPD Management With Novel Biologic Therapies}

Our understanding of the underlying pathophysiology of asthma and COPD has evolved significantly over the past decade. We have come to appreciate how heterogeneous these entities are, how many different cellular elements are involved, how many different cytokines and mediators are implicated, and how dynamic these diseases may be from one point in time to another. One broad way of classifying endotypes is based on activation of specific cytokines. Allergens generally cause activation of T helper cell type 2 (Th2) and innate lymphoid cells that activate IL-4, IL-5, and IL-13 to activate eosinophils and mast cells among others; this is referred to as type 2 inflammation. In contrast, viruses, bacteria, and irritants generally activate non-type 2 cells, including Th17 cells, that activate neutrophils through IL-6 and IL-17, among others. This new understanding has led to the development of precision interventions with biologic therapies that target specific elements of disease pathophysiology. Below are some of the pathways and new targeted therapies that are emerging for patients with asthma and COPD.

\section{Current Targets}

\section{IgE}

Inhaled allergens stimulate the production of $\operatorname{IgE}$ by $\mathrm{B}$ lymphocytes. B lymphocytes differentiate into plasma cells (the $\varepsilon$-switch), which produce and release IgE antibodies into circulation. IgE circulates in the blood, eventually binding to high-affinity $\operatorname{IgE}$ receptors on mast cells in tissue or peripheral blood basophils. When patients reencounter offending allergens, binding of the allergen with $\mathrm{IgE}$ induces the release of inflammatory mediators, such as histamines or leukotrienes, and which leads to the bronchoconstriction associated with asthma exacerbations.

Omalizumab was the first monoclonal antibody cleared for the management of asthma. Omalizumab binds to IgE, which inhibits the binding of $\operatorname{IgE}$ to the high-affinity $\operatorname{IgE}$ receptor on mast cells and basophils. This leads to reduced binding of $\operatorname{IgE}$ on these cells, which limits the degree of release of mediators of the allergic response. Omalizumab was cleared for use in patients age $>12$ y in $2003^{7,8}$ with allergic asthma (and subsequently down to age $6 \mathrm{y}$ ), after it was demonstrated to significantly reduce asthma exacerbations in patients with allergic asthma that was poorly controlled, despite the use of inhaled corticosteroids, and, subsequently, was demonstrated to reduce exacerbations in patients with more severe disease in patients on inhaled corticosteroids and long-acting $\beta$ agonists. Omalizumab is dosed based on IgE levels and body weight, and is administered every 2-4 weeks. More recent studies have shown that omalizumab has its greatest efficacy in patients with type 2 inflammation as characterized by elevated levels of blood eosinophils, periostin, or exhaled nitric oxide. ${ }^{9} \mathrm{Al}-$ though it is generally well tolerated, omalizumab injections can cause local pain at the injection site, and, rarely, hypersensitivity reactions. Omalizumab is not indicated for COPD.

\section{IL-5}

IL-5 is a cytokine that regulates proliferation, maturation, migration, and effector functions of eosinophils. It is produced by several cells, including Th2 lymphocytes, innate lymphoid cells, natural killer cells, eosinophils, basophils, and CD34+ cells. IL-5 messenger ribonucleic acid is increased in patients with asthma, is correlated with asthma severity, and is inducible by allergen exposure. Eosinophils have been implicated in both asthma and COPD, and contribute to airway inflammation through IL-5-mediated release of a variety of different granular proteins, including major basic protein, eosinophilic cationic protein, and eosinophil-derived neurotoxin. Because approximately half of patients with asthma and one third of the patients with COPD demonstrate airway eosinophilia that is associated with disease severity, IL-5 has become a major target for both asthma and COPD, ${ }^{10}$ and, currently, 3 biologics that target IL- 5 or its receptor have been cleared by the FDA.

Although early trials of mepolizumab, a monoclonal antibody that targets the IL-5 ligand, were successful in decreasing eosinophil counts, mepolizumab initially was not demonstrated to improve lung function across broad asthma populations. ${ }^{11,12}$ However, in trials that evaluated patients with sputum or blood eosinophilia, despite highdose inhaled corticosteroid or prednisone use, mepolizumab caused a significant decrease in exacerbations when compared with placebo. ${ }^{13-15}$ Mepolizumab was cleared in 2015 in patients with eosinophilic asthma ages $\geq 12 \mathrm{y}$.

Reslizumab, another monoclonal antibody that targets IL-5, is administered intravenously ( $3 \mathrm{mg} / \mathrm{kg}$ ) on a monthly 


\section{Biologic Therapies For Asthma AND COPD}

basis and has been shown to have significant effects not only on asthma symptoms but also on lung function and exacerbations when compared with placebo. ${ }^{16}$ Reslizumab was cleared in 2016 for patients $\geq 18$ y with eosinophilic asthma.

Benralizumab, a monoclonal antibody that targets IL$5 \mathrm{R} \alpha$, was cleared for use in eosinophilic asthma in 2017. In early studies, benralizumab was shown to decrease blood, sputum, and airway eosinophils, and, more recently, was also demonstrated to reduce asthma exacerbations, improve lung function, and reduce systemic corticosteroid dosing by as much as $75 \% .{ }^{17-20}$ One advantage that benralizumab has is that it may be administered subcutaneously every $8 \mathrm{wk}$ after the first 3 monthly doses. In addition to blocking the IL-5 receptor and preventing the IL-5 ligand from binding and activating the eosinophil, it also may get bound to natural killer cells, which may attack the eosinophils and result in eosinophilic depletion.

\section{Anti-IL-5 and COPD}

As of early 2018, no anti-IL-5 therapies have been cleared for use in COPD. However, 2 mepolizumab phase 3 studies showed improvements in exacerbation frequency in subjects treated with mepolizumab who had an eosinophilic phenotype and a history of COPD exacerbations, despite triple therapy. ${ }^{21}$ In the METREX study, ${ }^{21}$ among subjects with an eosinophilic phenotype (ie, $\geq 150$ cells $/ \mu \mathrm{L}$ at screening or $\geq 300$ cells $/ \mu \mathrm{L}$ anytime during the previous year), those treated with mepolizumab $100 \mathrm{mg}$ once monthly had a significantly lower annual moderate-to-severe exacerbation rate compared with subjects treated with placebo (1.40 vs $1.71, P=.04)$. In the METREO study, the annual exacerbation rate was not significantly different for the mepolizumab $100 \mathrm{mg}$, mepolizumab $300 \mathrm{mg}$, and placebo treatment groups $(1.19,1.27$, and 1.49 , respectively). These results indicate that there is likely some role for eosinophils in COPD but that more study is needed.

Although reslizumab has yet to be formally evaluated in clinical trials for COPD, in a phase 2 trial in subjects with COPD with sputum eosinophilia $(\geq 3 \%)$, benralizumab treatment did not significantly reduce the annual rate of moderate or severe exacerbations. ${ }^{22}$ However, significant improvements in $\mathrm{FEV}_{1}$ were observed in the overall population, and the results of prespecified subgroup analyses by baseline blood eosinophil count with benralizumab versus placebo have led to ongoing phase 3 trials to evaluate benralizumab in COPD.

\section{Future Targets}

\section{IL-4/IL-13}

IL-4 and IL-13 are cytokines that are found in increased levels in the airways and sputum of subjects with asthma.
IL-4 is produced primarily by Th2 cells and mast cells, whereas IL-13 is produced by Th2 cells, mast cells, eosinophils, and basophils. IL-4 and IL-13 share a common receptor, IL-4R $\alpha$, and act through the STAT 6 signaling pathway. IL-4 promotes Th2 cell development and B-cell isotype switching, and affects chemokine production by airway epithelium. IL-13 has effects on hematopoietic cells, airway epithelium, smooth muscle, fibroblasts, and the endothelium, which promote the allergic phenotype.

Dupilumab, a monoclonal antibody that inhibits the IL4R $\alpha$ subunit, was cleared in 2017 for treatment of atopic dermatitis and is being evaluated by the FDA for use in asthma. Dupilumab was superior to placebo in preventing asthma exacerbations and in improving lung function in patients receiving long-acting $\beta$ agonist and inhaled steroids, with or without blood or sputum eosinophilia. ${ }^{23,24}$

Lebrikizumab and tralokinumab, monoclonal antibodies that target IL-13, initially yielded benefits with respect to airway function when compared with placebo in patients with uncontrolled asthma, despite inhaled steroid use in some patients with high periostin or dipeptidyl peptidase- 4 levels. However, neither of these therapies was shown to be beneficial in phase 3 studies in targeted populations and are no longer being pursued in asthma. ${ }^{25,26}$ Dupilumab, lebrikizumab, and tralokinumab have not been extensively studied in COPD.

\section{Thymic Stromal Lymphopoietin and IL-33}

Thymic stromal lymphopoietin, a relatively novel target for asthma and COPD, is an epithelial-cell-derived cytokine that drives allergic inflammatory responses by activating dendritic cells and mast cells. Tezepelumab (AMG 157), a humanized monoclonal antibody that binds thymic stromal lymphopoietin, was evaluated in a phase 2 randomized double-blind placebo-controlled trial over a 52week treatment period. ${ }^{27}$ Three different doses of tezepelumab resulted in annualized asthma exacerbation rates at week 52 that were lower by 61,71 , and $66 \%$ compared with the placebo group $(P<.001$ for all comparisons $) .{ }^{27}$ Prebronchodilator $\mathrm{FEV}_{1}$ at week 52 was also higher in all tezepelumab groups compared with placebo. ${ }^{27}$ Overall, subjects treated with tezepelumab had lower rates of asthma exacerbations compared with those who received placebo, independent of baseline blood eosinophil counts, exhaled nitric oxide levels, or Th2 status. ${ }^{27}$ Anti-IL-33 therapies are currently in development, but, similar to anti-thymic stromal lymphopoietin, target many of the inflammatory pathways that are involved in activation of many of the cytokines noted above.

\section{Non-Th2 Inflammation Targets}

All of the cleared biologics and most of the biologics currently in development focus on Th2 inflammatory pathways. 


\section{Biologic Therapies for Asthma AND COPD}

This has left a large unmet need for patients with severe asthma without significant Th2 inflammation. IL-6 and IL-17 may promote both Th2 and non-type 2 inflammatory cascades; this makes these 2 cytokines targets of interest in mixed inflammatory obstructive airways diseases.

\section{IL-17}

IL-17, a cytokine produced by Th17 cells, plays an important role in the immunologic responses seen in asthma. Increased quantities of IL-17 have been isolated in blood, sputum, and human airway cells of patients with asthma. IL-17 receptor activation leads to the secretion of several inflammatory mediators, including IL- $1 \beta$, IL- 6 , tumor necrosis factor alpha, and granulocyte-macrophage colonystimulating factor, which ultimately leads to neutrophil recruitment. Brodalumab is a human monoclonal antibody that binds IL-17RA, which inhibits signaling of IL-17 and IL-25. In a phase $2 \mathrm{a}$ trial of adults with moderate-to-severe asthma, a prespecified subgroup that demonstrates high bronchodilator reversibility reported improved asthma control questionnaire scores, ${ }^{28}$ but no other clinically meaningful differences were found between the brodalumab groups and placebo, and this therapy has not been pursued further in asthma or COPD.

\section{CXCR2 and CCR3}

CXCR2 is a potent chemo-attractant for neutrophils that is being investigated in asthma and COPD. CXCR2 antagonists decrease IL-8 levels and have shown promise in early human trials. ${ }^{29}$ Navarixin, a CXCR2 receptor antagonist, reduced sputum and blood neutrophils and trend toward better asthma control based on Asthma Control Questionnaire (ACQ) but no significant change in $\mathrm{FEV}_{1} \cdot{ }^{30}$ An antisense oligonucleotide CCR3 antagonist (co-administered with an antisense oligonucleotide that targets the $\beta c$ subunit of the IL-3, IL-5, and granulocyte-macrophage colony-stimulating factor receptors) has shown some efficacy in phase 2 trials, decreasing sputum eosinophils in response to allergen challenge.

\section{Tyrosine Kinases}

Stem cell factor and its receptor, KIT, are central to mast cell homeostasis. Imatinib is a KIT inhibitor that was recently evaluated in a randomized double-blind placebocontrolled multicenter 24-week trial in subjects with severe asthma with airway hyper-responsiveness and poor asthma control despite maximum medical therapy. ${ }^{31}$ Imatinib inhibited mast cells and reduced airway hyper-responsiveness compared with placebo. ${ }^{31}$ These results indicated that KIT-dependent processes contribute to pathobiologic processes in severe asthma and may be appropriate targets for treatment of this disease. ${ }^{31}$ Further studies of imatinib in asthma are currently being planned, and other tyrosine kinase inhibitors that may affect both airway inflammation and remodeling are being tested in animal models and in early clinical trials.

\section{Summary and Future Directions}

We are in an exciting era in the management of asthma and COPD. We recognize that asthma and COPD are heterogeneous entities, but we are gaining a better understanding of underlying disease mechanisms and are using biomarkers and endotypes to personalize our approach to therapy. The new biologics are generally safe and well tolerated, but expensive, and it remains unclear to physicians and payers how to choose between therapies that may benefit patients with similar response profiles. It also remains unclear whether some patients may benefit from combinations of biologics that may affect different pathways that are simultaneously active in a given patient. Although the era of biologics has emerged in asthma, the focus has been on type 2 inflammation, whereas the absence of non-type 2 targeted therapies remains an unmet need. Novel targeted biologic therapies are not cleared in COPD, but the horizon is bright, with many studies ongoing. Although long-term safety studies and real-world efficacy studies are certainly warranted, the new biologics offer an opportunity to provide precision therapies that will improve outcomes in patients with asthma and COPD for years to come.

\section{REFERENCES}

1. Hekking PP, Wener RR, Amelink M, Zwinderman AH, Bouvy ML, Bel EH. The prevalence of severe refractory asthma. J Allergy Clin Immunol 2015;135(4):896-902.

2. National Asthma E, Prevention Program. Expert Panel Report 3 (EPR3): Guidelines for the Diagnosis and Management of Asthma-summary report 2007. J Allergy Clin Immunol 2007;120(5 Suppl):S94S138.

3. Global Initiative for Asthma (GINA). Global strategy for asthma management and prevention 2017. http://www.ginasthma.org/. Accessed March 29, 2018.

4. Global Initiative for Chronic Obstructive Lung Disease (GOLD). Global strategy for the diagnosis, management and prevention of COPD. 2017.

5. Chung KF, Wenzel SE, Brozek JL, Bush A, Castro M, Sterk PJ, et al. International ERS/ATS guidelines on definition, evaluation and treatment of severe asthma. Eur Respir J 2014;43(2):343-373.

6. Olin JT, Wechsler ME. Asthma: pathogenesis and novel drugs for treatment. BMJ 2014;349:g5517.

7. Busse W, Corren J, Lanier BQ, McAlary M, Fowler-Taylor A, Cioppa GD, et al. Omalizumab, anti-IgE recombinant humanized monoclonal antibody, for the treatment of severe allergic asthma. J Allergy Clin Immunol 2001;108(2):184-190.

8. Solèr M, Matz J, Townley R, Buhl R, O’Brien J, Fox H, et al. The anti-IgE antibody omalizumab reduces exacerbations and steroid requirement in allergic asthmatics. Eur Respir J 2001;18(2):254-261.

9. Hanania NA, Wenzel S, Rosén K, Hsieh HJ, Mosesova S, Choy DF, et al. Exploring the effects of omalizumab in allergic asthma: an analysis of biomarkers in the EXTRA study. Am J Respir Crit Care Med 2013;187(8):804-811. 


\section{Biologic Therapies For Asthma AND COPD}

10. Tashkin DP, Wechsler ME. Role of eosinophils in airway inflammation of chronic obstructive pulmonary disease. Int J Chron Obstruct Pulmon Dis 2018;13:335-349.

11. Flood-Page P, Swenson C, Faiferman I, et al. A study to evaluate safety and efficacy of mepolizumab in patients with moderate persistent asthma. Am J Respir Crit Care Med. 2007;176(11):10621071.

12. Kiele MJ, ten Brinke A, Khan J, et al. Effects of an interleukin-5 blocking monoclonal antibody on eosinophils, airway hyperresponsiveness, and the late asthmatic response. Lancet. 2000;356(9248): 2144-2148.

13. Pavord ID, Korn S, Howarth P, Bleecker ER, Buhl R, Keene ON, et al. Mepolizumab for severe eosinophilic asthma (DREAM): a multicentre, double-blind, placebo-controlled trial. Lancet 2012; 380(9842):651-659.

14. Ortega HG, Liu MC, Pavord ID, Brusselle GG, FitzGerald JM, Chetta A, et al.; MENSA Investigators. Mepolizumab treatment in patients with severe eosinophilic asthma. N Engl J Med 2014;371(13):11981207.

15. Bel EH, Wenzel SE, Thompson PJ, Prazma CM, Keene ON, Yancey SW, et al.; SIRIUS Investigators. Oral glucocorticoid-sparing effect of mepolizumab in eosinophilic asthma. N Engl J Med 2014;371(13): 1189-1197.

16. Castro M, Zangrilli J, Wechsler ME, Bateman ED, Brusselle GG, Bardin P, et al. Reslizumab for inadequately controlled asthma with elevated blood eosinophil counts: results from two multicentre, parallel, double-blind, randomised, placebo-controlled, phase 3 trials. Lancet Respir Med 2015;3(5):355-366.

17. Castro M, Wenzel SE, Bleecker ER, Pizzichini E, Kuna P, Busse WW, et al. Benralizumab, an anti-interleukin 5 receptor $\alpha$ monoclonal antibody, versus placebo for uncontrolled eosinophilic asthma: a phase $2 \mathrm{~b}$ randomised dose-ranging study. Lancet Respir Med 2014; 2(11):879-890.

18. Bleecker ER, FitzGerald JM, Chanez P, Papi A, Weinstein SF, Barker $\mathrm{P}$, et al.; SIROCCO study investigators. Efficacy and safety of benralizumab for patients with severe asthma uncontrolled with high-dosage inhaled corticosteroids and long-acting $\beta 2$-agonists (SIROCCO): a randomised, multicentre, placebo-controlled phase 3 trial. Lancet 2016; 388(10056):2115-2127.

19. FitzGerald JM, Bleecker ER, Nair P, Korn S, Ohta K, Lommatzsch M, et al.; CALIMA study investigators. Benralizumab, an anti-interleukin-5 receptor $\alpha$ monoclonal antibody, as add-on treatment for patients with severe, uncontrolled, eosinophilic asthma (CALIMA): a randomised, double-blind, placebo-controlled phase 3 trial. Lancet 2016;388(10056):2128-2141

20. Nair P, Wenzel S, Rabe KF, Bourdin A, Lugogo NL, Kuna P, et al.; ZONDA Trial Investigators. Oral glucocorticoid-sparing effect of benralizumab in severe asthma. N Engl J Med 2017;376(25):24482458.

21. Pavord ID, Chanez P, Criner GJ, Kerstjens HAM, Korn S, Lugogo $\mathrm{N}$, et al. Mepolizumab for eosinophilic chronic obstructive pulmonary disease. N Engl J Med 2017;377(17):1613-1629.

22. Brightling CE, Bleecker ER, Panettieri RA, et al. Benralizumab for chronic obstructive pulmonary disease and sputum eosinophilia: a randomised, double-blind, placebo-controlled, phase 2A study. Lancet Respir Med. 2014;2(11):891-901.

23. Wenzel S, Ford L, Pearlman D, Spector S, Sher L, Skobieranda F, et al. Dupilumab in persistent asthma with elevated eosinophil levels. N Engl J Med 2013;368(26):2455-2466.

24. Wenzel S, Castro M, Corren J, Maspero J, Wang L, Zhang B, et al. Dupilumab efficacy and safety in adults with uncontrolled persistent asthma despite use of medium-to-high-dose inhaled corticosteroids plus a long-acting $\beta 2$ agonist: a randomised double-blind placebocontrolled pivotal phase $2 \mathrm{~b}$ dose-ranging trial. Lancet 2016; 388(10039):31-44.

25. Hanania NA, Korenblat P, Chapman KR, Bateman ED, Kopecky P, Paggiaro $\mathrm{P}$, et al. Efficacy and safety of lebrikizumab in patients with uncontrolled asthma (LAVOLTA I and LAVOLTA II): replicate, phase 3, randomised, double-blind, placebo-controlled trials. Lancet Respir Med 2016;4(10):781-796.

26. Brightling CE, Chanez P, Leigh R, O'Byrne PM, Korn S, She D, et al. Efficacy and safety of tralokinumab in patients with severe uncontrolled asthma: a randomised, double-blind, placebo-controlled, phase 2b trial. Lancet Respir Med 2015;3(9):692-701.

27. Corren J, Parnes JR, Wang L, Mo M, Roseti SL, Griffiths JM, et al. Tezepelumab in adults with uncontrolled asthma. N Engl J Med 2017;377(10):936-946.

28. Busse WW, Holgate S, Kerwin E, Chon Y, Feng J, Lin J, Lin SL. Randomized, double-blind, placebo-controlled study of brodalumab, a human anti-IL-17 receptor monoclonal antibody, in moderate to severe asthma. Am J Respir Crit Care Med 2013;188(11):1294-1302.

29. Nair P, Gaga M, Zervas E, et al. Safety and efficacy of a CXCR2 antagonist in patients with severe asthma and sputum neutrophils: a randomized, placebo-controlled clinical trial. Clin Exp Allergy. 2012; 42(7):1097-1103

30. Nair P, Gaga M, Zervas E, Alagha K, Hargreave FE, O'Byrne PM, et al. Safety and efficacy of a CXCR2 antagonist in patients with severe asthma and sputum neutrophils: a randomized, placebo-controlled clinical trial. Clin Exp Allergy 2012;42(7):1097-1103.

31. Cahill KN, Katz HR, Cui J, Lai J, Kazani S, Crosby-Thompson A, et al. KIT inhibition by imatinib in patients with severe refractory asthma. N Engl J Med 2017;376(20):1911-1920.

\section{Discussion}

Strange: I would make a cautionary note on your search for specific genotypes or endotypes. In our alpha-1 antitrypsin experience, in which we know the gene, sometimes the genotype gives you a patient with asthma, bronchiectasis, emphysema, and sometimes it isn't all in the genes. So, sticking with phenotyping might be the way to go.
Wechsler: There's been a huge effort to try to identify specific genetic predictors of responsiveness to different asthma therapies. With the $\beta$ agonists, we talked about the arg/arg genotype, and there are also pharmacogenetics looking at inhaled steroids. It might be that we need to identify specific responders to therapies because of their cost, by doing pharmacogenetics. But I still feel like pharmacogenetics is somewhat in its infancy despite the fact that there have been thousands of papers published, $95 \%$ of which are review articles as opposed to substantive articles that have changed practice.

Peters: So you brought up the point that, when you look at mepolizumab studies, the reduction of exacerbations is probably a key feature of these therapies. When you actually look at just emergency department visits and hospitalizations rather than lumping oral corticosteroids in, how effective are- 


\section{Biologic Therapies For Asthma AND COPD}

they? In looking at the original 2 studies in mepolizumab, ${ }^{1,2}$ when they evaluated a broad section of subjects with asthma rather than subjects with severe asthma with frequent exacerbations, they really could not show significant changes in quality of life or symptom control. How much data are there for the monoclonal antibodies other than omalizumab that document quality of life and reduction in symptoms? When Busse et $\mathrm{al}^{3}$ tried to stop omalizumab in their study, they reported that their subjects relapsed. That has not been our experience in San Antonio; we have been able to taper some patients off omalizumab and followed up on them to document stability. Do you feel that once you start patients on these monoclonal therapies that you are expecting to keep them on lifelong?

Wechsler: I'll address the second question first, which is the issue of how long should we treat these patients. These drugs have only been available, the anti-IgE has been around for a while, and people either respond or do not respond to these therapies. There have been some studies that show that, if you stop the omalizumab, then there is actually an increase in exacerbations in those who had been responding if they stop it. The same thing has been demonstrated with the anti-IL-5 therapies. If you stop it, within 3 or 4 months, then there is an increase in exacerbations. There have been some open-label extension studies that have demonstrated that, in people who were responding to therapy and then came off therapy slowly, they had an increase in their eosinophils over a few months and they had increased risks of exacerbations over that time. The first question was?

Peters: When you omit the studies that include exacerbations defined by the need for oral corticosteroids and look at reductions in severe exacerbations (requiring emergency depart- ment visits or hospitalizations), do these therapies still show efficacy?

Wechsler: Right, so if you want to look at just emergency department visits and hospitalizations are much less common than asthma exacerbations. And asthma exacerbations are defined as $\geq 3$ d of systemic corticosteroids for increased symptoms of asthma. Some people look at asthma deterioration. That's another definition of asthma exacerbations as sort of a composite end point of deterioration. We've looked at that as well. ${ }^{4}$ Emergency department visits and hospitalizations are much less common; they're $<5-10 \%$ of all exacerbations. However, with each of these biologics, if you do a subgroup analysis, there's a reduction in those patients by approximately $70 \%$ with reslizumab and mepolizumab. With regard to benralizumab, there's a benefit there as well. ${ }^{5}$ Again, those events are much less common, but there is a significant impact on those outcomes. Frankly that's what payers are most interested in. They're interested in those outcomes in specific, and there's a 50$70 \%$ reduction with different monoclonals as well as with bronchial thermoplasty, by the way. ${ }^{6}$

Lugogo: I think the vast majority of prescribers of omalizumab are actually allergists. Furthermore, most of the drug is prescribed by a small percentage of the total population. So, for instance, at our institution, there might be 1 or 2 clinicians who use biologics and then there are 40 other clinicians who almost never give any patient biologics. Apart from encouraging phenotyping and things like that, I wonder how we can encourage our fellow pulmonologists to prescribe these medications to patients with severe asthma. This is similar to my comments about low utilization of alpha-1 replacement therapy. Of course, in contrast, biologic drugs in asthma have been shown to have profound effects. In spite of this, I think there is a lot of resistance to giving very expensive therapies to patients particularly because oral corticosteroids are cheap. There is also this idea that patients with asthma who are not well controlled are somewhat to blame because they are not adherent and don't take their inhaled medications. This has resulted in a mental block when it comes to taking up new therapies. Therefore, how should we as providers who are leading the charge in terms of the care of patients with asthma address this so there is some uptake? Because there are some patients who would really benefit from these therapies who are not receiving them at this time.

Wechsler: Looking at some of the prescription data, only approximately $10-15 \%$ of pulmonologists prescribe biologics for asthma. Obviously none for COPD because it isn't cleared for COPD as of yet. Now a part of it is the heterogeneity of pulmonologists, some just do critical care, some just do pulmonary fibrosis, and some just do lung cancer. You wouldn't expect those people to prescribe mepolizumab or reslizumab for asthma. But most physicians out there in a clinical practice, they do see asthma. Part of the problem is logistics. When omalizumab came out, most pulmonologists were not set up to give shots. They weren't set up in their regular clinic to store the drug, prepare the drug, and things like that. So there tended to be a huge referral to allergists who are use to giving allergy shots on a regular basis. That being said, with these new therapies coming out, I think we have to educate physicians, pulmonologists, allergists, even primary care physicians that there are other options and that these are quite effective in a subset of patients, and that patients who are on systemic corticosteroids, in particular, I view it unacceptable for people to be systematic corticosteroids in this day and age. I think it's our job, and part of what we're doing here is to educate respiratory therapists about what is out there 
but also primary care physicians so they say, "Okay, there are these newer therapies that are expensive but they have a significant impact in terms of pharmacoeconomics but, most importantly, reduction in adverse effects of systemic corticosteroids, and also improvement in quality of life and efficacy". Many of the studies that have been done have demonstrated those benefits as well, and I think that's the most important thing. Most of our patients who are on these therapies and continue on them do really well and like being on them. It can be somewhat of a nuisance. Hopefully, over time, it will result in drugs that are given every 8 weeks or maybe home injectables so they won't need to come into the office. I think we need to educate about efficacy of these therapies. It's also important to advocate for reduction in cost with the pharmaceutical industry. Although the sticker price is $\$ 32,000 /$ year, most insurers or payers don't pay that, they broker some kind of a deal. So that may make more pharmacoeconomic sense.

MacIntyre: From a practical perspective, say you have a really tough patient with asthma who's been difficult to control on routine medications. What's the Wechsler protocol for assessing biomarkers and making decisions?

Wechsler: The first thing I do is an extensive workup; make sure they have asthma. Part of that extensive workup that isn't done most places is that we do bronchoscopies on those patients with severe conditions to try to identify whether they have some kind of infection going on, whether they have vocal cord dysfunction, whether they have significant gastroesophageal or laryngopharyngeal reflux, and we try to treat those things. But while we're doing that workup we also include blood eosinophils and nitric oxide, and check their IgE level. In some patients, if their phenotype is suggestive, we will also do cystic fi- brosis and alpha-1 antitrypsin testing, and we have identified several patients who had unexpected alpha-1 antitrypsin deficiency by doing that kind of testing in our cohort. That would be the first step. Some places do a lot more sputum, like McMaster University in Hamilton, Ontario and University of California, San Francisco. Then, I will try to identify what the predominant biomarker that's going. Do they have high eosinophils versus low eosinophils? I'll also use a little of their phenotypic characteristics because reslizumab is an intravenous preparation that's dosed in $\mathrm{mg} / \mathrm{kg}$, and, in the patients who is more obese, I might give reslizumab instead of mepolizumab. We'll see when benralizumab comes out but, hopefully, that will be an effective therapy that can be given every 8 weeks, which some patients might prefer as well, and, if a patient has low eosinophils and low nitric oxide, then I am less likely to use one of these therapies. There is a paucity of therapies, but it's possible that, in those patients, right now I might do bronchial thermoplasty, if they have no inflammation, no eosinophils, low IgE, and low nitric oxide. Maybe when dupilumab comes out, it will be an effective therapy for those patients, in addition to the patients with type- 2 inflammation with eosinophilia.

George: I think it's really interesting when the payers are saying that, in some respects, they want to withhold these costly therapies from patients because they have been non-adherent to other treatments. But there is nothing that will quickly sort out which patients are truly motivated enough and warrant some kind of biologic therapy. I think about patients I've treated with anti-IgE coming in twice a month, and, each time they come, they get 3 injections based on this chart, and someone does that every other week for years on end, I think it's a really quick point to make about trying to separate out the patients who really are non-adherent versus those who are motivated to do something to get better.

Wechsler: What's been shown is that those who respond to these therapies tend to stay adherent. And if they stop being adherent, they feel miserable and go back. Another point is that there was one study, published by Busse et al, ${ }^{7}$ that shows that, for some therapies, such as anti-IgE, it might be beneficial to give it seasonally during allergy season or at the beginning of school and that might be just as good in that cohort. It was an innercity asthma study that demonstrated that. I agree with you, and we'll see with some of the therapies, like benralizumab, when that comes out at every 8 weeks, how adherent do you really need to be? With the dupilumab study I showed you, the subjects could actually stop their inhaled steroids long-acting $\beta$ agonists and still had an $87 \%$ reduction in their exacerbations. ${ }^{8}$ So, this could have a significant impact, particularly in those patients who are non-adherent. You're getting these therapies and they're not every day but every 2, 4, or 8 weeks might be beneficial, particularly if there is home administration.

\section{REFERENCES}

1. Nair P, Pizzichini MM, Kjarsgaard M, Inman MD, Efthimiadis A, Pizzichini E, et al. Mepolizumab for prednisone-dependant asthma with sputum eosinophilia. N Engl J Med 2009;360(10):985-993.

2. Haldar P, Brightling CE, Hargadon B, Gupta S, Monteiro W. Mepolizumab and exacerbations of refractory eosinophilic asthma. N Engl J Med 2009;360(10):973-984.

3. Ledford D, Busse W, Trzaskoma B, Omachi TA, Rosén K, Chipps BE, et al. A randomized multicenter study evaluating Xolair persistence of response after long-term therapy (XPORT). J Allergy Clin Immunol 2017; 140(1):162-169.e2.

4. Wechsler ME, Yawn BP, Fuhlbrigge AL, Pace WD, Pencina MJ, Doros G, et al. Anticholinergic vs. long-acting $\beta$-agonist in combination with inhaled corticosteroids in black adults with asthma: the BELT randomized clinical trial. JAMA 2015;314(16): 1720-1730. 


\section{Biologic Therapies For Asthma AND COPD}

5. Nair P, Wenzel S, Rabe KF, Bourdin A, Lugogo NL, Kuna P, et al.; ZONDA Trial Investigators. Oral glucocorticoid-sparing effect of benralizumab in severe asthma. N Engl J Med 2017;376(25):2448-2458.

6. Wechsler ME, Laviolette M, Rubin AS, Fiterman J, Lapa e Silva JR, Shah PL, et al.; Asthma Intervention Research 2 Trial Study Group. Bronchial thermoplasty: Long-term safety and effectiveness in severe persistent asthma. J Allergy Clin Immunol 2013;132(6):1295-1302.

7. Busse WW, Morgan WJ, Gergen PJ, Mitchell HE, Gern JE, Liu AH, et al. Rancomized trial of omalizumab (anti-IgE) for asthma in inner-city children. N Engl J Med 2011; 364(11):1005-1015.

8. Wenzel S, Ford L, Pearlman D, Spector S, Sher L, Skobieranda F, et al. Dupilumab in persistent asthma with elevated eosinophil levels. N Engl J Med 2013;368(26):2455-2466.

This article is approved for Continuing Respiratory Care Education credit. For information and to obtain your CRCE

(free to AARC members) visit www.rcjournal.com 